\title{
Systems Biology Ontology: Update
}

\author{
Nick Juty, EMBL-EBI
}


- Brief introduction SBO

- annotation layer issue

- reiterate / clarify the problem

- describe resolution process

- OBO Foundry Status

- what it is, and how to get it

- Relationship between Systems Biology Graphical Notation (SBGN) with SBO

- new glyphs 


\section{http://www.ebi.ac.uk/sbo/}

- Provide a strictly defined relational vocabulary of terms for use in Systems Biology

- A navigable structure of terms that has 'parents', 'children' 
6 orthogonal vocabularies:

- entity (macromolecule)

- interaction (transport, reactions)

- mathematical expression (mass action rate law)

- modelling framework (discrete)

- participant roles (S, P, M)

- quantitative parameters (Hill coefficient) 


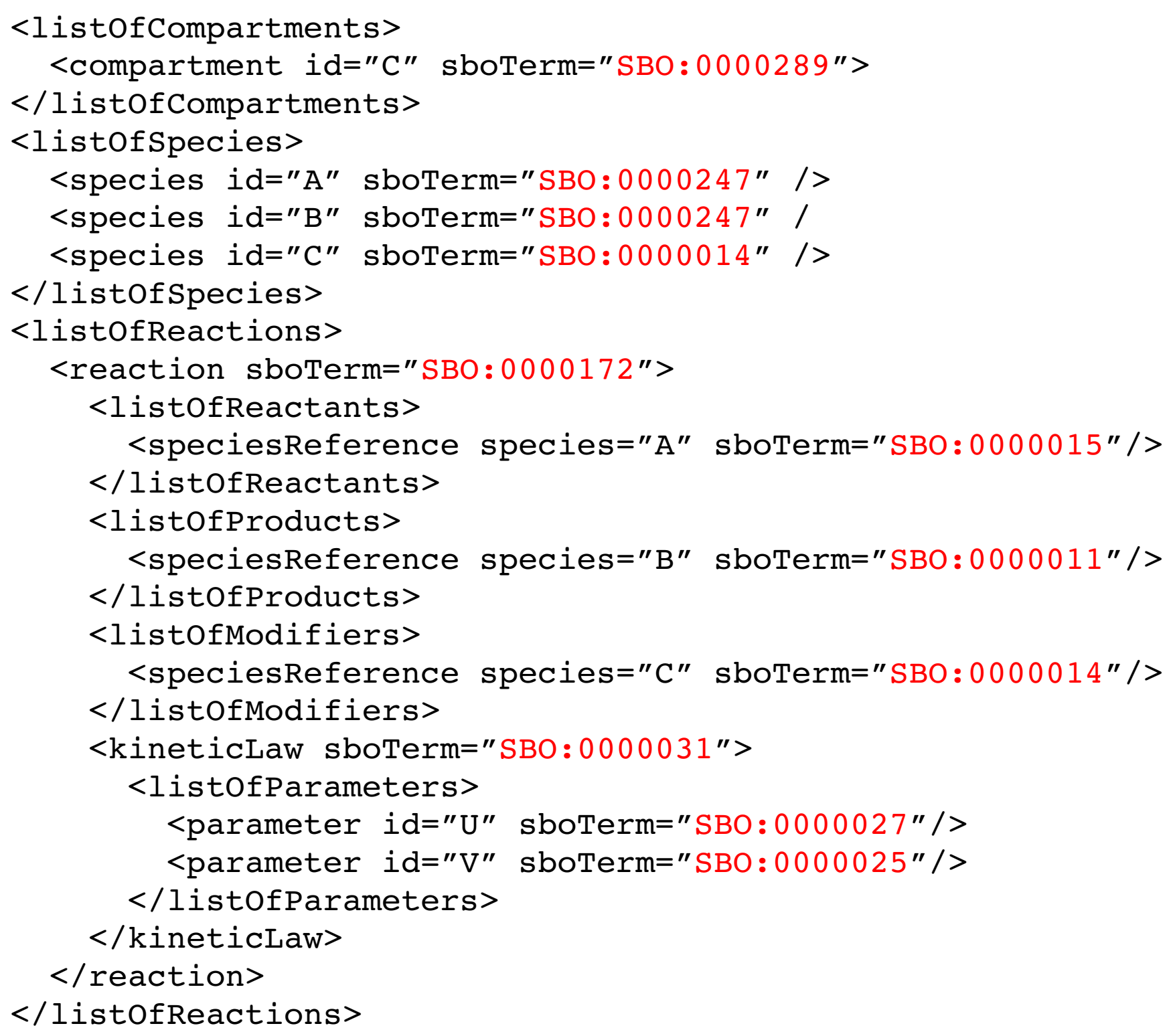




\section{Semantic layer:}

- link between models encoded in SBML and graphical notations (such as SBGN)

- conversion to semantically enriched computing formats (such as BioPAX)

- translation of models between continuous deterministic frameworks and discrete stochastic framework

- merging of models 
SBO annotation layer (as it was):

SBML component

\section{SBO branch}

<speciesReference>

participant role

<parameter>

quantitative parameter

$<$ kineticLaw>

rate law

<Species>

entity 
SBO annotation layer (as it was):

\section{SBML component}

<speciesReference>

<parameter>

..

$<$ kineticLaw>

<Species>
EXAMPLE

substrate inhibitor

kinetic constant time

mass action rate law

simple chemical enzyme

\section{SBO branch}

participant role

quantitative parameter

rate law

entity 
SBO annotation layer

(as it was):

SBML component

SBO branch

<speciesReference>

participant role

<kineticLaw>

rate law 
SBO annotation layer (as it was):

SBML component

SBO branch

<speciesReference> participant role

$<$ kineticLaw> rate law

$<$ bvar $>$

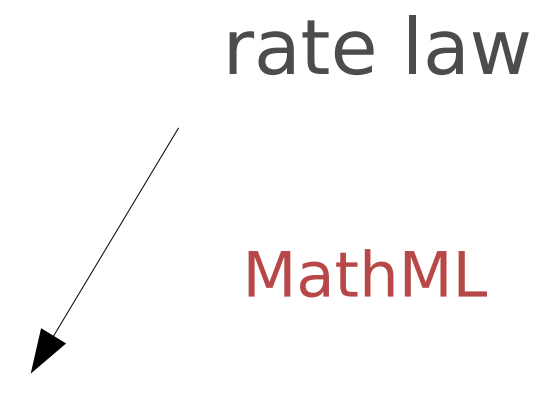

$<$ ci definitionURL="http://biomodels.net/SBO/\#SBO:0000015"> substrate <.. $<c$ i definitionURL="http://biomodels.net/SBO/\#SBO:0000011" > product $<$. .. 
SBO annotation layer
(as it was):
SBML component

\section{SBO branch}

<speciesReference> $\quad$ substrate $\quad$ product participant role

$<$ kineticLaw $>$

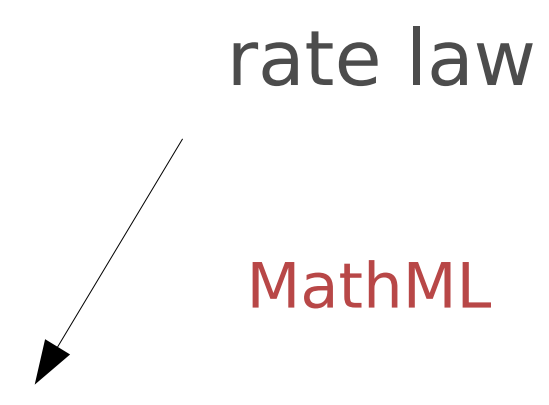

<bvar>

SBO:0000015" > substrate <..

SBO:0000011" $>$ product $<$..

COMBINE 2010

Edinburgh, $6^{\text {th }}-9^{\text {th }}$ October 
Problem: 'substrate' (participant role) = amount? concentration?

กั

$\overleftarrow{\circ}$

\section{SBML component}

\author{
SBO branch
}

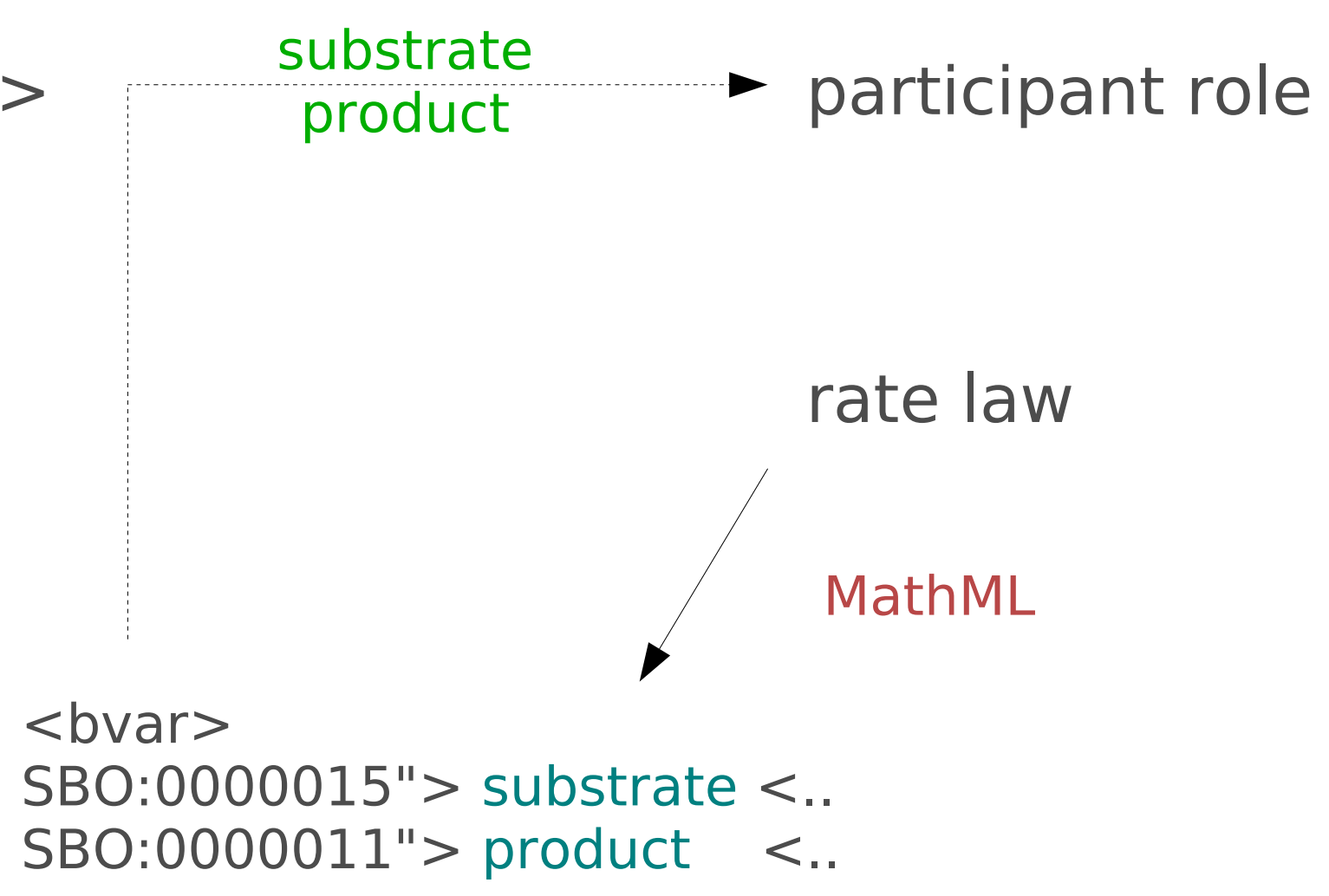

COMBINE 2010

Edinburgh, $6^{\text {th }}-9^{\text {th }}$ October 
- Initial changes made on SBO 'demo'

- Introduce 'quantitative parameter's for 'participant role' terms

\section{quantitative parameter}

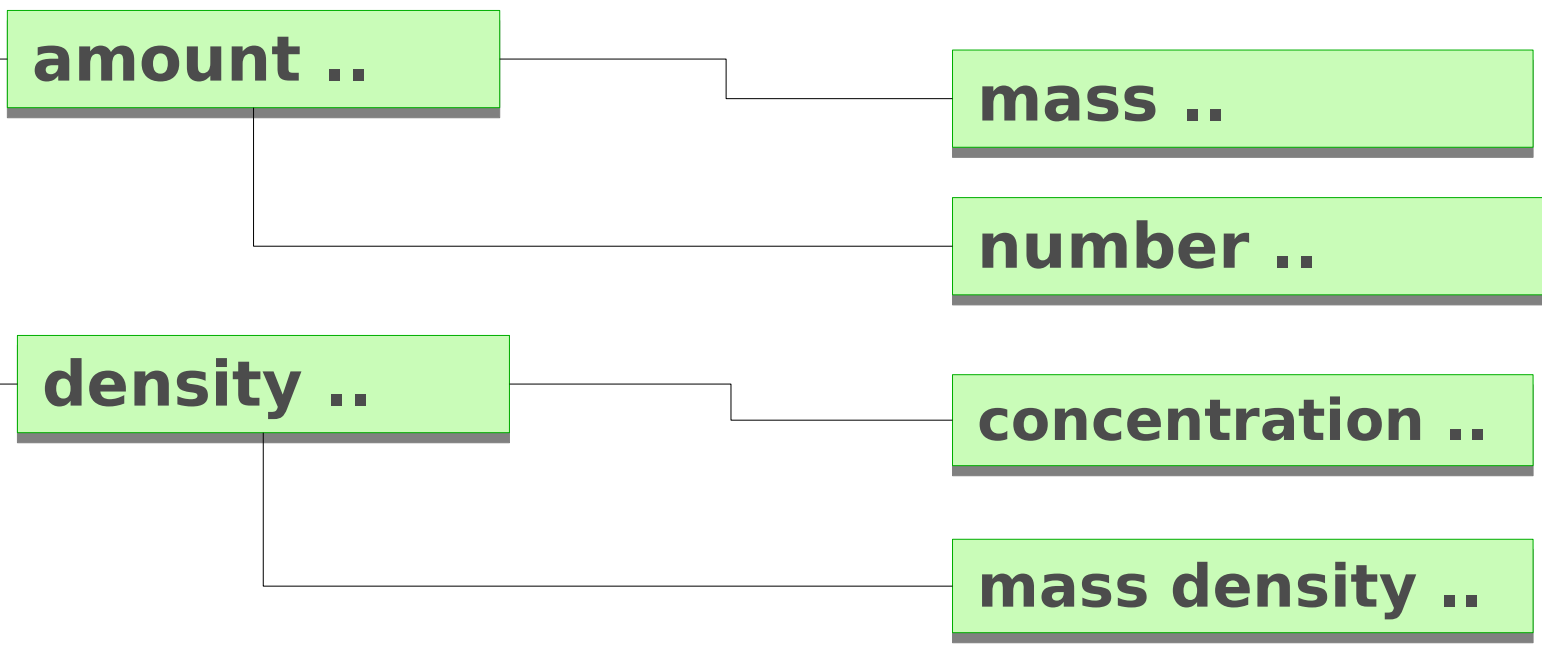

- Modify <bvar> links in all MathML - redirect to quantitative parameter branches

- Request feedback for selected users (validate)

- Move changes to SBO 'main' (implement $\rightarrow$ live version)

COMBINE 2010

Edinburgh, $6^{\text {th }}-9^{\text {th }}$ October 
- Open Biomedical ontologies - open community-level collaborative ontology development

- First Principles:

- open - terms should be available for use without restriction

- changeable - modified by community effort

- orthogonal - additivity and modularity

- machine friendly - interpretable and syntactically sound

- identifiers - backward compatible

- >80 library members, 8 Foundry 


\section{http://www.obofoundry.org/}

\section{$\Delta$ The Open Biomedical Ontologies}
Ontologies
Resources
Participate
About

The $\mathrm{OBO}$ Foundry is a collaborative experiment involving developers of science-based ontologies who are establishing a set of principles for ontology development with the goal of creating a suite of orthogonal interoperable reference ontologies in the biomedical domain. The groups developing ontologies who have expressed an interest in this goal are listed below, followed by other relevant efforts in this domain.

In addition to a listing of $\mathrm{B} \bigcirc$ ontologies, this site also provides a statement of the $\bigcirc \mathrm{BO}$ Foundry principles, discussion fora, technical infrastructure, and other services to facilitate ontology development. We welcome feedback and encourage participation.

Click any column header to sort the table by that column. The fis link to the term request trackers for the listed ontologies.

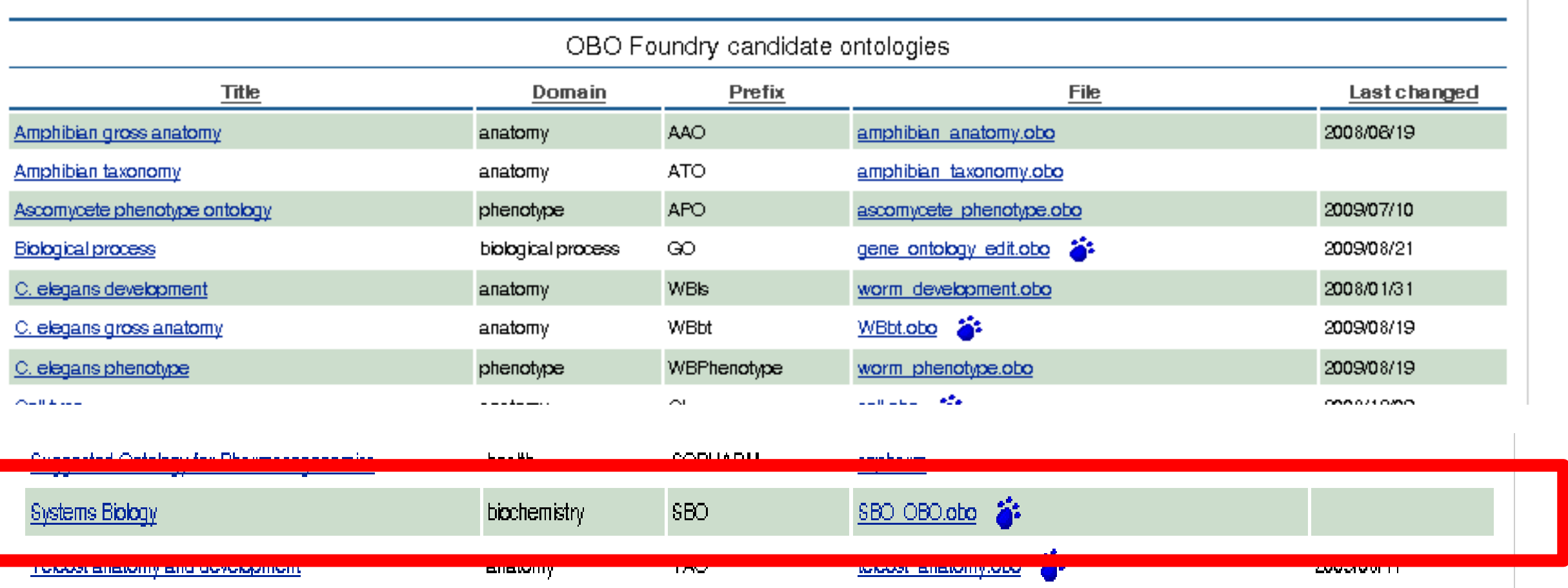

COMBINE 2010

Edinburgh, $6^{\text {th }}-9^{\text {th }}$ October 
- SBO is an OBO candidate ontology

- Review $15^{\text {th }}$ Feb 2010

- Issues to address include:

- Expose MathML terms (hidden relationships)

- Versioning

- Uniqueness/orthogonality

- MIREOT (direct import)

- Cross reference

- Cross products

- Interface:

- References for term (creation)

- Cross references (relationships to other ontologies) 


\section{SBGN}

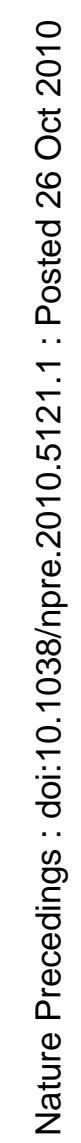

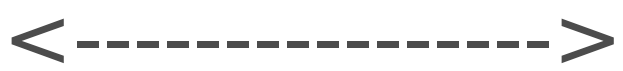

Process (PD)

Stimulation (ER)

And (AF)
SBO

SBO:0000375

SBO:0000170

SBO:0000173 


\section{SBO / SBGN metadata}

\section{SBGN}

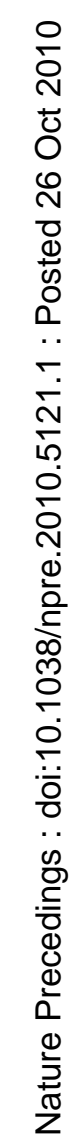

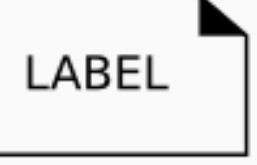

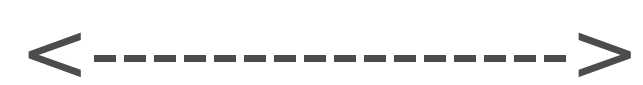

(ER)

\section{Annotation}

SBO

$?$

- Do we need a $7^{\text {th }}$ branch in SBO to accommodate:

- Annotation,

- cross-references,

- metadata 


\section{Acknowledgements}

\section{EBI}

Mélanie Courtot

Camille Laibe

Nicolas Le Novère

Lukas Endler

Karim Tazibt

SBML team

Michael Hucka

Sarah Keating
BioModels Database developers and curators

bbsrc

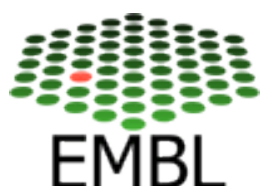

engMS

ELIXIR 


\section{EBI}

Mélanie Courtot

Camille Laibe

Nicolas Le Novère

Lukas Endler

Karim Tazibt

SBML team

Michael Hucka

Sarah Keating
BioModels Database developers and curators

\section{The community of Systems Biology for their contributions, their software support and their comments.}

https://sourceforge.net/projects/sbo
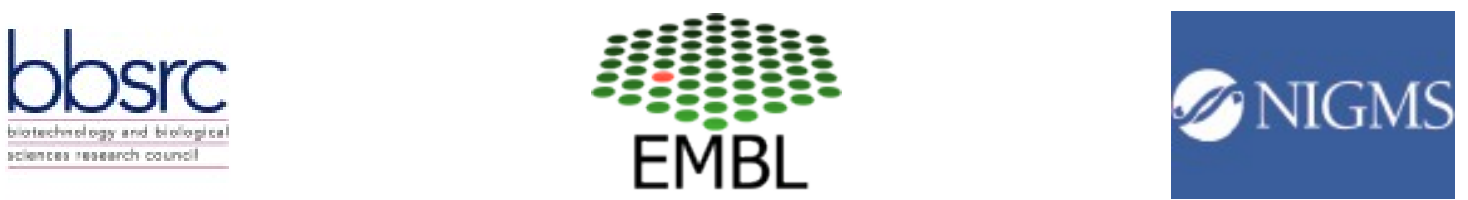